\title{
CALCULATING FLOAT IN LINEAR SCHEDULES WITH SINGULARITY FUNCTIONS
}

\author{
Gunnar Lucko \\ Angel A. Peña Orozco \\ Department of Civil Engineering \\ The Catholic University of America \\ 620 Michigan Avenue NE \\ Washington, DC 20064, U.S.A.
}

\begin{abstract}
This paper presents an exact approach of calculating float for each activity in linear schedules. It is based on singularity functions, which have been used previously to determine the criticality of activities in said schedules. Singularity functions are versatile in that they can describe multiple changes of productivity within each activity, can be evaluated by hand if desired, and thus provide the basis for a complete schedule analysis methodology. Following a brief review of how activities and their buffers are modeled with singularity functions, this paper examines types of float that are commonly encountered in the critical path method of scheduling and develops an equivalent approach for linear schedules. An example from the literature is used to demonstrate the application of the new float analysis.
\end{abstract}

\section{INTRODUCTION}

Linear scheduling concepts are found under a variety of names in the literature (Harris and Ioannou 1998). In general, the term linear scheduling as used in this paper shall refer to scheduling activities in a two-dimensional coordinate system, where one axis shows time. Activities follow an order of precedence along this axis. Buffers determine how far apart activities have to remain, Other constraints may also apply, e.g. fixed milestones for individual activities or the overall project. The second axis in the diagram of a linear schedule shows the amount of work that has been produced during a specific amount of time, measured in a unit that is chosen to be reflect the nature of the work. It can be geometric, e.g. the length of roadway paved, volumetric, e.g. the volume of concrete that has been placed, cardinal, e.g. the number of apartments that have been painted, ordinal, e.g. the floors of a building that have been outfitted, financial, e.g. the dollar value of different materials that have been installed, or be unitless, e.g. the percentage of work that has been completed to date. It is important that all activities share the same amount unit.
The literature provides examples of two different axis arrangements. Whether the time or the amount axis is vertical often depends on the horizontal or vertical direction of the actual work. In the diagrams of this paper the time axis is shown vertically to represent how activities "stack up" over time as they are added to the schedule. Note that the slope of each line in the diagram is the inverse of its productivity, which is defined as work amount over time (Harmelink and Rowings 1998). Various types of construction projects are presented in the literature and analyzed with linear scheduling, including those with a geometric linearity, e.g. road building (Arditi and Albulak 1986) and highrise building (O'Brien 1975), and those with repetitive operations, e.g. floors of high-rise buildings (Thabet and Beliveau 1994), which may overlap with the first type and lend themselves to simulation. Linear schedules can identify potential conflicts where lines touch or cross in the diagram. Changing the productivity of activities, postponing their starts, or re-sequencing resolves such conflicts.

\subsection{Time and Amount Buffers}

Buffers are used to specify exactly how close activities may come (Arditi et al. 2002). As presented in this paper, each buffer follows an activity to which it is attributed, but the method can also model buffers that precede an activity as used by Kallantzis and Lambropoulos (2004). Note that since buffers are modeled with separate equations they can take on shapes that are independent of their host activities. Buffers can act into either of the two axis directions of the linear schedule (Harmelink and Rowings 1998). These constraints shall be called time buffer and amount buffer (instead of the less generic term location buffer), respectively. A time buffer demands that a successor may only start after a particular duration has passed since its predecessor, akin to a lead time on a link under the critical path method (CPM). An amount buffer demands that a successor may only start after a particular distance is gained to its predecessor, which has no equivalent concept under CPM. 


\section{Lucko and Peña Orozco}

\section{SINGULARITY FUNCTIONS}

Lucko (2007b) has presented an integrated mathematical modeling approach for linear schedules that uses singularity functions, which had originally been used by Macaulay (1919) and Föppl (1927) for structural engineering analysis. Equation 1 contains their elementary term, which is written with pointed brackets. It links a function with a value at which it become valid, akin to an on / off switch.

$$
\langle x-a\rangle^{n}=\left\{\begin{array}{c}
0 \text { for } x<a \\
(x-a)^{n} \text { for } x \geq a
\end{array}\right.
$$

where $x$ is the variable on the horizontal $x$-axis, $a$ is the cutoff value on the $x$-axis at which the function becomes valid, and $n$ is the order of the phenomenon. Equation 1 is zero for all $x<a$, and is evaluated normally for all $x \geq a$. For example, $n=0$ gives a step function at $a, n=1$ gives a ramp function growing from $a$, and $n=2$ gives a quadratic curve growing from $a$. The desired shape is gained by multiplying Equation 1 with a scaling factor $m$, which becomes e.g. the step height for $n=0$ or the ramp slope for $n=1$.

Complex shapes of $y(x)$ are created by superposition of any number of elementary terms, which yields the full singularity function. Each change in the behavior of $y(x)$ thus necessitates adding one change term to the singularity function, which is evaluated cumulatively - with growing value of $x$, more terms become active. If a term is active only for a range $\left\{a_{1}\right.$ to $\left.a_{2}\right\}$ it must first be added with one term at $a_{1}$ and then deducted again at $a_{2}$ with another term.

\subsection{General Model}

Using the notation of Equation 1, the activities and buffers of a linear schedule are described with one singularity function each as per the general model given in Equation 2. As written, it assumes straight segments in the schedule, i.e. piecewise constant productivities, but can also be generalized to any higher order, e.g. to model learning effects.

$$
\begin{aligned}
& y(x)=\Delta t_{0} \cdot\langle x-0\rangle^{0}+\frac{\Delta t_{1}}{\Delta a_{1}} \cdot\langle x-0\rangle^{1}+\left[\frac{\Delta t_{2}}{\Delta a_{2}}-\frac{\Delta t_{1}}{\Delta a_{1}}\right] \cdot\left\langle x-\Delta a_{1}\right\rangle^{1} \\
& +\left[\frac{\Delta t_{3}}{\Delta a_{3}}-\frac{\Delta t_{2}}{\Delta a_{2}}\right] \cdot\left\langle x-\left(\Delta a_{1}+\Delta a_{2}\right)\right\rangle^{1}+\ldots
\end{aligned}
$$

where $y$ is the time variable, $x$ is the amount variable, $\Delta t_{\mathrm{j}}$ are durations on the $y$-axis, and $\Delta a_{\mathrm{j}}$ are ranges on the $x$-axis. The first term contains the intercept $t_{0}$, followed by the initial slope $\Delta t_{1} / \Delta a_{1}$, and change terms in rectangular brackets thereafter that add a new slope and deduct the old slope. It is recommended to simplify all ratios to the smallest non-common numerators and denominators and to sort the terms by cutoff values $a$ and within that by order $n$.

\subsection{Criticality Analysis}

An essential part of analyzing linear schedules is to determine the equivalent of a critical path. The reader is referred to Lucko (2007a) for an in-depth discussion of the following steps of the analytical algorithm. An important result of that study was the distinction between time criticality and amount criticality, which are caused by the respective buffer types. It is further possible that activities are only partially critical, whereby non-critical segments may occur at either or both the starting or the finishing end of an activity. This creates up to three different segments whose criticality differs within each activity (Harmelink 2001).

1. Activities: Activities are listed with their order of precedence, productivity and changes thereof, time and / or amount buffers, and any other constraints in the schedule.

2. Initial Equations (Stacking): Singularity functions are written, alternating activities and buffers such that the maximum of all predecessors is the intercept of the successor, similar to CPM with only finish-to-start links. In case of constant buffer values, the time buffer equation can be generated by adding the time buffer to the intercept of the host activity equation and the amount buffer equation can be generated by deducting the buffer value from all cutoff values, i.e. a shift to the negative direction on the $x$-axis.

3. Differences: Difference equations between neighboring activities and buffers are written for each link in the order of precedence. Elementary terms of the same cutoff value $a$ and order $n$ can be added to simplify the equations.

4. Differentiation: The differences are differentiated as per Equation 3 and evaluated for $x$-values where the sign changes cumulatively from negative to positive. Minimum differences between activities occur at these critical points.

$\frac{d}{d x}\langle x-a\rangle^{n}=n \cdot\langle x-a\rangle^{n-1}$

5. Final Equations (Consolidation): The minimum differences are deducted cumulatively from the intercepts of the activities and buffers in the order of precedence. Other mathematical constraints can be included here. This generates the singularity functions for the linear schedule that has been consolidated to its minimum overall duration.

6. Criticality: The critical path is obtained by connecting the overall start and finish of the linear schedule via critical points and marking critical activity segments. Note that separate time and amount critical paths may exist depending on the buffer types. Critical activity segments are always connected across a buffer parallel to the direction of the axis into which the particular buffer type acts. 


\section{Lucko and Peña Orozco}

\section{FLOAT ANALYSIS}

Harris and Ioannou (1998) presented a small construction schedule and analyzed its float in Awwad and Ioannou (2007). Table 1 lists its six activities and their durations per one unit of work, and successors. Activity $A$ achieves only half the normal productivity for units 3 and 4 . Activity $B$ is interrupted for 5 days between units 3 and 4 . Activity $C$ skips work on unit 5. A finish-to-start link connects the first unit of activity $A$ with $C$ via a lead time of two days. An amount buffer of one unit is assessed for each activity.

Table 1: Activities

\begin{tabular}{|c|c|c|c|}
\hline Activity & $\begin{array}{c}\text { Unit } \\
\text { Duration }\end{array}$ & Successors & Production \\
\hline $\mathrm{A}$ & $2 d$ & $\mathrm{~B}, \mathrm{C}$ & $\begin{array}{l}\text { Half productivity } \\
\text { for units } 3 \text { and } 4 \\
\text { After unit } 1 \text {, } \\
\text { finish-to-start lead } \\
\text { time of } 2 \text { days to } C\end{array}$ \\
\hline B & $1 d$ & $\mathrm{D}$ & $\begin{array}{l}5 \text { days interruption } \\
\text { between units } 3 \text { and } 4\end{array}$ \\
\hline $\mathrm{C}$ & $4 d$ & $\mathrm{D}, \mathrm{E}$ & $\begin{array}{l}\text { No work on unit } 5 \text {, } \\
\text { but still continuous }\end{array}$ \\
\hline $\mathrm{D}$ & $3 d$ & $\mathrm{~F}$ & Regular activity \\
\hline E & $1 d$ & $\mathrm{~F}$ & Regular activity \\
\hline $\mathrm{F}$ & $1 d$ & - & Regular activity \\
\hline
\end{tabular}

Equations 4 through 9 list the activities in their final consolidated form. Amount buffer equations are omitted for brevity but can be easily derived. Note that no amount buffer exists for activity $F$, because it is the last activity.

$$
\begin{aligned}
& y(x)_{A}=0 \cdot\langle x-0\rangle^{0}+\frac{2}{1} \cdot\langle x-0\rangle^{1}+\frac{2}{1} \cdot\langle x-2\rangle^{1}-\frac{2}{1} \cdot\langle x-4\rangle^{1} \\
& y(x)_{B}=9 \cdot\langle x-0\rangle^{0}+\frac{1}{1} \cdot\langle x-0\rangle^{1}+5 \cdot\langle x-3\rangle^{0} \\
& y(x)_{C}=2 \cdot\langle x-0\rangle^{0}+\frac{4}{1} \cdot\langle x-0\rangle^{1}-\frac{4}{1} \cdot\langle x-4\rangle^{1}+\frac{4}{1} \cdot\langle x-5\rangle^{1} \\
& y(x)_{D}=11 \cdot\langle x-0\rangle^{0}+\frac{3}{1} \cdot\langle x-0\rangle^{1} \\
& y(x)_{E}=17 \cdot\langle x-0\rangle^{0}+\frac{1}{1} \cdot\langle x-0\rangle^{1} \\
& y(x)_{F}=24 \cdot\langle x-0\rangle^{0}+\frac{1}{1} \cdot\langle x-0\rangle^{1}
\end{aligned}
$$

\subsection{Critical Path Method}

Two types of float are typically calculated during a CPM analysis. The free float (FF) of an activity is determined as the difference between the minimum earliest start (ES) of all successors and the earliest finish (EF) of the current activity. FF indicates how much the current activity can be delayed without impacting any successors. The total float (TF) of an activity is the difference between the latest start (LS) and the ES of said activity. It can also be calculated as the difference between the latest finish (LF) and the LS. TF indicates how much the current activity can be delayed without impacting the overall project finish. Figure 1 shows the two float types for the gray middle activity, whose extent is represented by arrows. The abbreviations indicate if activities are in their earliest or latest constellation. Links to other activities in the larger schedule that caused the particular configurations are omitted for clarity.

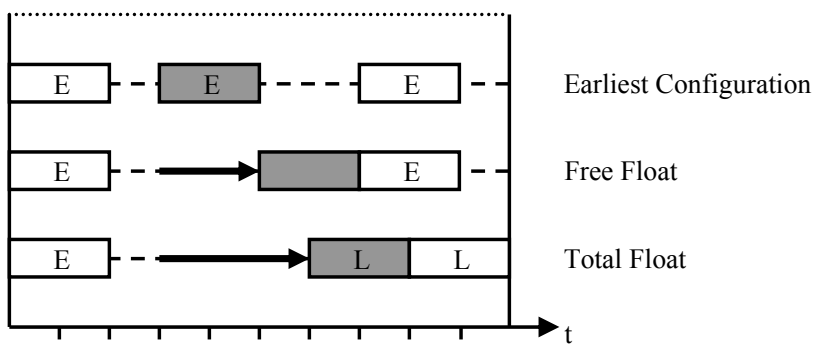

Figure 1: Float Types from Critical Path Method

\subsection{Linear Scheduling}

Harmelink (2001, p255f., emphasis in original) defined rate float as "the amount that the production rate of an noncontrolling linear activity can be lowered before the activity will become a controlling segment". Rate float is a change in productivity, measured in amount per time, and is consumed by a rotation or shift of non-critical activity segments. Ammar (2003) also used time float, measured in time units, which as consumed by a shift parallel to the time axis and calculated as the difference between either the starts or the finishes of an activity and its direct successor. It was further distinguished into total float and free float as under CPM. This definition only assessed float at individual points, not over a continuous range. Awwad and Ioannou (2007) calculated total float for a linear schedule with and without enforcing resource continuity and rate float, but also limited their model to individual points.

For activities of constant productivity, it is useful to distinguish divergence and convergence, which can assist in determining the potential float. Unless they are parallel, two activities will either create a start-to-start link if they diverge or a finish-to-finish link if they converge (Harris and Ioannou 1998). In general, float occupies blank areas around the non-critical activity segments, into which they could rotate to consume it. For converging activities the float of the successor shrinks toward the critical point, which shall be called early float. For diverging activities the float of the predecessor grows beyond the critical point, which shall be called late float. Of course, complex schedules whose activities change their productivity require an exact mathematical analysis to determine the actual float. 


\section{Lucko and Peña Orozco}

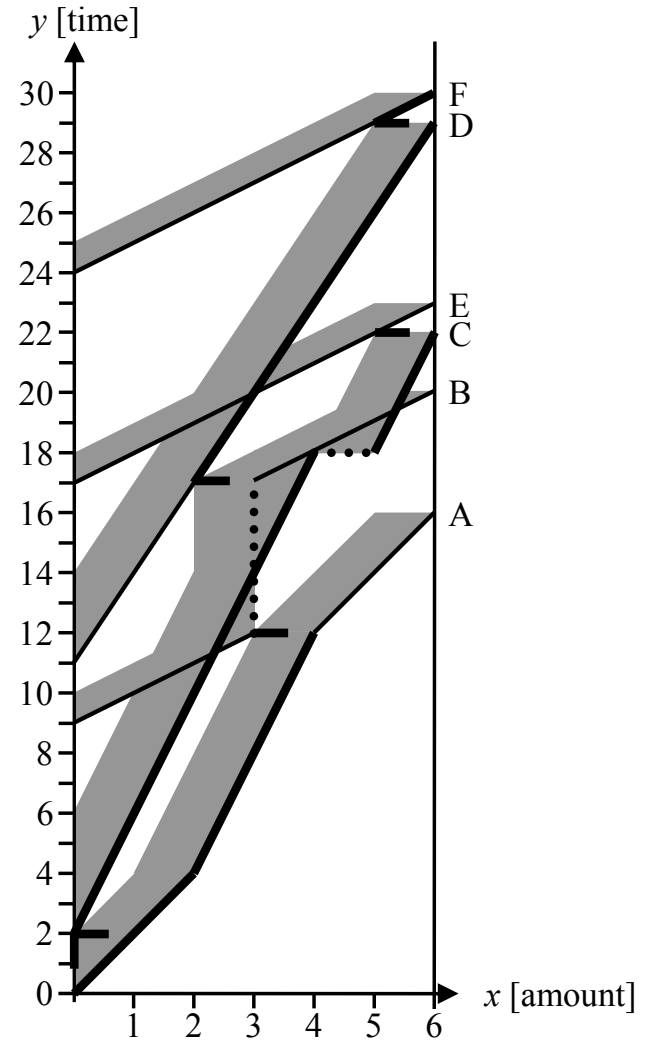

Figure 2: Free Float Critical Path

\subsection{Example Calculations}

Following the criticality analysis as described earlier and distinguishing further between criticality based on FF versus criticality based on TF yields the ranges as per Table 2 .

Table 2: Criticality

\begin{tabular}{ccc}
\hline & \multicolumn{2}{c}{ Amount Range } \\
\cline { 2 - 3 } Activity & Free Float & Total Float \\
\hline A & $0 u$ to $4 u$ & $0 u$ to $4 u$ \\
B & Only at $3 u$ & Only at $3 u$ \\
C & $0 u$ to $6 u$ & No \\
D & $2 u$ to $6 u$ & $2 u$ to $6 u$ \\
E & No & No \\
F & $5 u$ to $6 u$ & $5 u$ to $6 u$ \\
\hline & &
\end{tabular}

Figures 2 and 3 show these FF and TF critical paths as thick solid lines. Both are amount criticalities. Only amount buffers exist in the example, which are shown as dark gray areas. Note in Table 2 that more activity segments are considered critical under the narrower definition of FF (delaying any successor already makes a segment critical) than under the wider definition of TF (only delaying the overall project finish makes a segment critical).

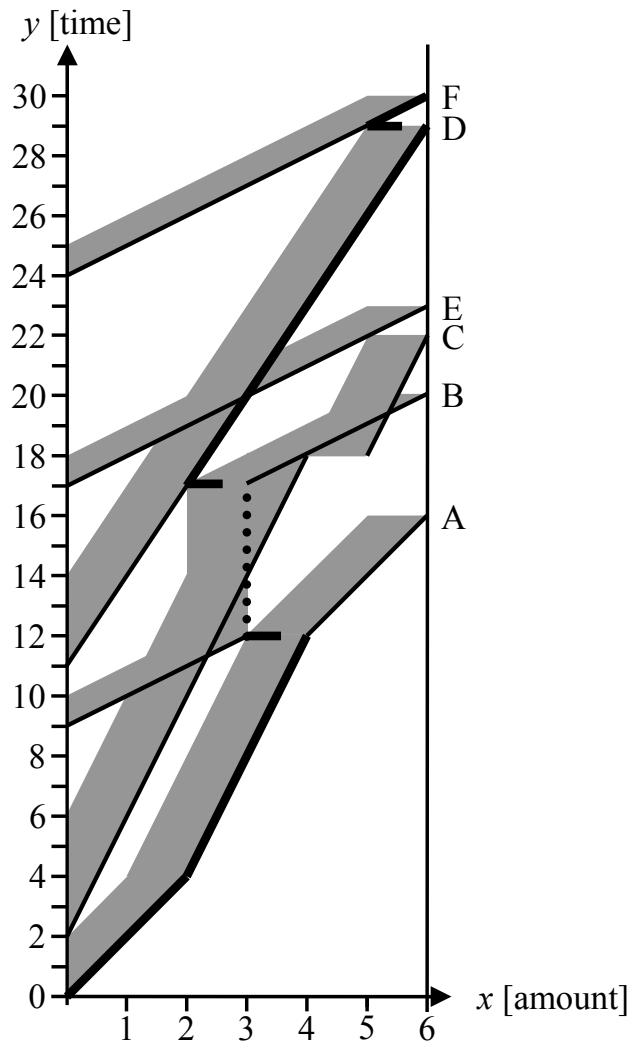

Figure 3: Total Float Critical Path

\subsubsection{Free Float Equations}

The float analysis presented in this paper assumes that only rotations of non-critical segments occur (Harmelink 2001). The more complex case of allowing interruptability of activities and shifting individual segments thereof to consume float will be the topic of future research. Free float equations are differences between equations of successor activities and predecessor buffers for each link in the order of precedence. These equations are only evaluated over non-critical segments, even if blank areas extend further.

\subsubsection{Late Free Float (Buffer of $A$ to $C$ )}

$y(x)_{C-b u f A}=0 \cdot\langle x-0\rangle^{0}+\left(\frac{4}{1}-\frac{2}{1}\right) \cdot\langle x-0\rangle^{1}-\frac{2}{1} \cdot\langle x-1\rangle^{1}$
$+\frac{2}{1} \cdot\langle x-3\rangle^{1}-\frac{4}{1} \cdot\langle x-4\rangle^{1}+\left(\frac{4}{1}+\frac{2}{1}\right) \cdot\langle x-5\rangle^{1}$

Late float is consumed by an upward rotation of noncritical segments. Equation 10 represents the entire "white area" between the buffer of $A$ and $C$. However, to fulfill the amount buffer constraint for late free float, a plateau must be reached prior to the finish of activities $A, B$, and $E$. 


\section{Lucko and Peña Orozco}

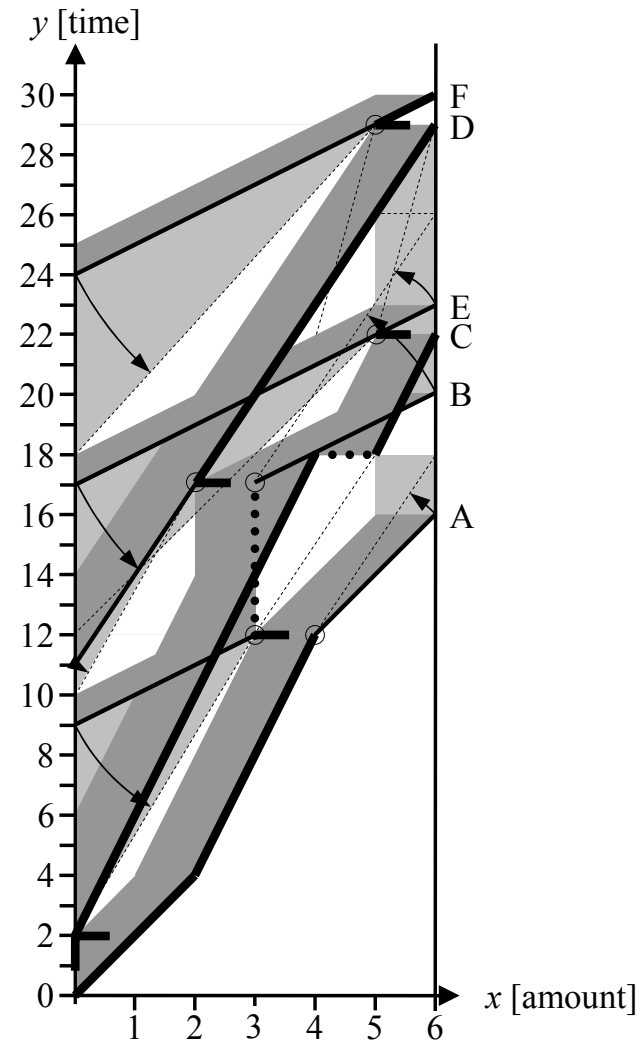

Figure 4: Free Float and Rate Float

At $x_{\max }=6 u$ minus the amount buffer the value is $y(5$ $u)_{C \text { - buf } A}=2 d$. Equation 10 must be modified for late float from the critical point onward. The possible slope of the free float over the non-critical segment $x=\{4 u$ to $6 u\}$ changes from $2 d / 1 u$ for $A$ to $3 d / 1 u$ for finishing $A$ late by $2 d$. This rotation is on the safe side and yields Equation 11. It gives the time of the free float at $x_{\max }=6 u$ as $2 d$. The rate float over $x=\{4 u$ to $6 u\}$ is the inverted and simplified difference of unsimplified slopes $1 u / 6 d$, to obey the arithmetic rule for ratio inversion that $a / b-c / d \neq b / a-$ $d / c$. Figure 4 shows the free float as light gray areas.

$y(x)_{C-b u f A}^{L F F}=0 \cdot\langle x-4\rangle^{0}+\left(\frac{3}{1}-\frac{2}{1}\right) \cdot\langle x-4\rangle^{1}$

\subsubsection{Early Free Float (B to Buffer of A)}

$y(x)_{B-b u f A}=7 \cdot\langle x-0\rangle^{0}+\left(\frac{1}{1}-\frac{2}{1}\right) \cdot\langle x-0\rangle^{1}-\frac{2}{1} \cdot\langle x-1\rangle^{1}+5 \cdot\langle x-3\rangle^{0}$

$+\frac{2}{1} \cdot\langle x-3\rangle^{1}+\frac{2}{1} \cdot\langle x-5\rangle^{1}$

Early float is consumed by a downward rotation of non-critical segments. Equation 12 gives the time of the free float at $x_{\min }=0 u$ as $7 d$. It represents the entire "white area" between the buffer of $A$ and $B$. It must be modified for early float prior to the critical point. The possible slope over $x=\{0 u$ to $3 u\}$ changes from $1 d / 1 u$ for $B$ to $10 d /$ $3 u$ for starting $B$ early by $7 d$. This rotation yields Equation 13. The rate float over $x=\{0 u$ to $3 u\}$ is $7 u / 10 d$.

$$
y(x)_{B-b u f A}^{E F F}=7 \cdot\langle x-0\rangle^{0}-\left(\frac{10}{3}-\frac{1}{1}\right) \cdot\langle x-0\rangle^{1}
$$

\subsubsection{Late Free Float (Buffer of B to D)}

Equation 14 is modified into 15 in analogy to modifying Equation 10 into 11 . It gives the time of the free float at $x_{\max }=6 u$ as $6 d$. The slope changes from $1 d / 1 u$ to $3 d / 1$ $u$. The rate float over $x=\{3 u$ to $6 u\}$ is $2 u / 3 d$.

$$
\begin{aligned}
& y(x)_{D-b u f B}=1 \cdot\langle x-0\rangle^{0}+\left(\frac{3}{1}-\frac{1}{1}\right) \cdot\langle x-0\rangle^{1}-5 \cdot\langle x-2\rangle^{0}+\frac{1}{1} \cdot\langle x-5\rangle^{1} \\
& y(x)_{D-b u f B}^{L F F}=0 \cdot\langle x-3\rangle^{0}+\left(\frac{3}{1}-\frac{1}{1}\right) \cdot\langle x-3\rangle^{1}
\end{aligned}
$$

\subsubsection{Early Free Float (D to Buffer of B)}

Equation 14 already describes this free float. It gives the time of the free float at $x_{\min }=0 u$ as $1 d$. It represents the entire "white area" between the buffer of $B$ and $D$. It must be modified for early float prior to the critical point. The possible slope over $x=\{0 u$ to $2 u\}$ changes from $3 d / 1 u$ for $D$ to $7 d / 2 u$ for starting $D$ early by $1 d$. This rotation yields Equation 16. The rate float over $x=\{0 u$ to $2 u\}$ is 1 $u / 21 d$.

$y(x)_{D-b u f B}^{E F F}=1 \cdot\langle x-0\rangle^{0}-\left(\frac{7}{2}-\frac{3}{1}\right) \cdot\langle x-0\rangle^{1}$

\subsubsection{Early Free Float (E to Buffer of C)}

Equation 17 gives the time of the free float at $x_{\min }=0 u$ as $11 d$. However, differentiation as per Equation 3 finds that the minimum distance of $2 d$ between $E$ and the buffer of $C$ over $x=\{0 u$ to $5 u\}$ occurs at $x=3 u$, where the cumulative sign of Equation 18 changes from negative to positive. The shift in $C$ reduces the available free float.

$y(x)_{E-b u f C}=11 \cdot\langle x-0\rangle^{0}+\left(\frac{1}{1}-\frac{4}{1}\right) \cdot\langle x-0\rangle^{1}+\frac{4}{1} \cdot\langle x-3\rangle^{1}-\frac{4}{1} \cdot\langle x-4\rangle^{1}$

$+\frac{4}{1} \cdot\langle x-5\rangle^{1}$

$y(x)_{E-b u f C}^{\prime}=0-\frac{3}{1} \cdot\langle x-0\rangle^{0}+\frac{4}{1} \cdot\langle x-3\rangle^{0}-\frac{4}{1} \cdot\langle x-4\rangle^{0}$

$+\frac{4}{1} \cdot\langle x-5\rangle^{0}$ 


\section{Lucko and Peña Orozco}

The $2 d$ at $x=3 u$ is extended proportionally over $x=$ $\{0 u$ to $5 u\}$, which gives the time of the free float at $x_{\min }=$ $0 u$ as $5 d$. Equation 17 is modified for early float prior to the critical point. The possible slope over $x=\{0 u$ to $5 u\}$ changes from $1 d / 1 u$ for $E$ to $2 d / 1 u$ for starting $E$ early by $5 d$. This rotation yields Equation 19. The rate float over $x=\{0 u$ to $5 u\}$ is $1 u / 2 d$.

$$
y(x)_{E-b u f C}^{E F F}=5 \cdot\langle x-0\rangle^{0}-\left(\frac{2}{1}-\frac{1}{1}\right) \cdot\langle x-0\rangle^{1}
$$

\subsubsection{Late Free Float (Buffer of $\mathbf{E}$ to $F$ )}

Equation 20 is modified into 21 in analogy to modifying Equation 10 into 11. It gives the time of the free float at $x_{\max }=6 u$ as $6 d$. The slope changes from $1 d / 1 u$ to $7 d / 1$ $u$. The rate float over $x=\{5 u$ to $6 u\}$ is $6 u / 7 d$.

$$
\begin{aligned}
& y(x)_{F-b u f E}=6 \cdot\langle x-0\rangle^{0}+\left(\frac{1}{1}-\frac{1}{1}\right) \cdot\langle x-0\rangle^{1}+\frac{1}{1} \cdot\langle x-5\rangle^{1} \\
& y(x)_{E-b u f F}^{L F F}=0 \cdot\langle x-5\rangle^{0}+\left(\frac{7}{1}-\frac{1}{1}\right) \cdot\langle x-5\rangle^{1}
\end{aligned}
$$

\subsubsection{Early Free Float (F to Buffer of E)}

Equation 20 already describes this free float. It gives the time of the free float at $x_{\text {min }}=0 u$ as $6 d$. It represents the entire "white area" between the buffer of $E$ and $F$. It must be modified for early float prior to the critical point. The possible slope over $x=\{0 u$ to $5 u\}$ changes from $1 d / 1 u$ for $F$ to $11 d / 5 u$ for starting $F$ early by $6 d$. This rotation yields Equation 22. The rate float over $x=\{0 u$ to $5 u\}$ is 6 $u / 11 d$.

$$
y(x)_{F-b u f E}^{E F F}=6 \cdot\langle x-0\rangle^{0}-\left(\frac{11}{5}-\frac{1}{1}\right) \cdot\langle x-0\rangle^{1}
$$

Table 3 lists the ranges of non-critical segments for the FF and TF critical paths of Figures 2 and 3 . Note that it is the remainder of Table 2, which lists critical segments. Table 4 lists the values for both time and rate float. Note that slope changes in Figure 4 are inversely related to productivity changes and thus are not graphically proportional.

Table 3: Float Ranges

\begin{tabular}{cccc}
\hline & \multicolumn{2}{c}{ Amount Range } & $\begin{array}{c}\text { Type of } \\
\text { Free Float }\end{array}$ \\
\cline { 2 - 3 } Activity & Free Float & Total Float & Free \\
\hline A & $4 u$ to $6 u$ & $4 u$ to $6 u$ & Late \\
B & $0 u$ to $6 u$ & $0 u$ to $6 u$ & Early, Late \\
C & No & $0 u$ to $6 u$ & - \\
D & $0 u$ to $2 u$ & $0 u$ to $2 u$ & Early \\
E & $0 u$ to $6 u$ & $0 u$ to $6 u$ & Early, Late \\
F & $1 u$ to $5 u$ & $1 u$ to $5 u$ & Early \\
\hline
\end{tabular}

Table 4: Maximum Free Float Values and Rate Float

\begin{tabular}{ccccc}
\hline & \multicolumn{2}{c}{ Early Float } & \multicolumn{2}{c}{ Late Float } \\
\cline { 2 - 5 } Activity & Time & Rate & Time & Rate \\
\hline A & - & - & $2 d$ & $1 / 6 u / d$ \\
B & $7 d$ & $7 / 10 u / d$ & $6 d$ & $2 / 3 u / d$ \\
C & - & - & - & - \\
D & $1 d$ & $1 / 21 u / d$ & - & - \\
E & $5 d$ & $1 / 2 u / d$ & $6 d$ & $6 / 7 u / d$ \\
F & $6 d$ & $6 / 11 u / d$ & - & - \\
\hline
\end{tabular}

Float equations can be evaluated for any $x$-value within the ranges of non-critical segments listed in Table 3 . Inserting $x=0$ or $x=6$, respectively, into the final equations for free float gives the maximum times as listed in Table 4. Note again that the early and late floats for each activity in Table 4 are derived from two separate equations.

\subsubsection{Total Float Equations}

Total float equations must be generated after the free float equations. Same as before, it is necessary to distinguish between early and late float. Early total float is calculated as the maximum sum of all free float equations that precede the early non-critical segment until the next critical activity or the first activity in the order of precedence. Note that early float means that an activity would start earlier than scheduled but progress at a lower productivity. Late total float is calculated as the maximum sum of all free float equations that succeed the late non-critical segment until the next critical activity or the last activity in the order of precedence. These equations must be evaluated over noncritical segments only. Only free float equations are presented in this paper for brevity. Additional total float equations can be derived in analogy to the previous sections.

\subsubsection{Comparison with Repetitive Scheduling Method}

Comparing the results from using singularity functions with the float analysis for the repetitive scheduling method (RSM) is possible for the case that the continuity of all activities is maintained. The total float critical path, called controlling sequence (Awwad and Ioannou 2007), differs between the two analyses, from $A-B-D-F$ with continuous buffers using singularity functions to $A-C-D-F$ with pointwise checking of minimum distances. The critical segments for the latter case ranged from $0 u$ to $1 u, 0 u$ to $4 u$, $3 u$ to $6 u$, and $5 u$ to $6 u$, respectively. Float times and rates are given as $4 d / 3 u$ for the first segment of $B$ starting at $y=6 d, 5 d / 3 u$ for the second one starting at $14 d$, $2 d / 1 u$ for the second segment of $C$ starting at $y=20 d$, and $4 d / 6 u$ for $E$ starting at $y=19 d$. The reason for the differences to the values of Tables 2 and 4 is that RSM does not assess amount buffer constraints continuously for 


\section{Lucko and Peña Orozco}

all $x$-values but only at so-called control points, which places activities $B, C$, and $E$ into different positions than when analyzed with singularity functions. Further research is necessary to fully examine the impact of such discrete versus continuous treatment of constraints. A direct comparison is also difficult because under RSM the non-critical segments with early float rotate around the start point of their activity, not around a critical point. The effects of such partial criticality were not examined further by RSM.

\section{CONCLUSIONS}

The float analysis with singularity functions has the potential to become an important tool for schedulers who wish to answer detailed questions about how delays in individual activities can be absorbed in linear schedules and how they may impact other activities. Float can be calculated exactly both in time units and as a change in productivity. It clearly distinguishes whether the criticality is created by time or amount buffers, whether free or total float occur in an activity segment, and whether the non-critical segment should rotate to start earlier or later to consume its float.

\section{REFERENCES}

Ammar, M. A. 2003. Float analysis of non-serial repetitive activities. Construction Management and Economics 21(5): 535-542.

Arditi, D., O. B. Tokdemir, K. Suh. 2000. Challenges in line-of-balance scheduling. Journal of Construction Engineering and Management 128(6): 545556.

Arditi, D., M. Z. Albulak. 1986. Line-of-balance scheduling in pavement construction. Journal of Construction Engineering and Management 112(3): 411-424.

Awwad, R. E., P. G. Ioannou. 2007. Floats in RSM: Repetitive scheduling method. Proceedings of the 2007 Construction Research Congress, eds. P. S. Chinowsky, A. D. Songer, P. M. Carrillo, Grand Bahama Island, The Commonwealth of the Bahamas, May 6-8, 2007, American Society of Civil Engineers, Reston, Virginia, $8 \mathrm{p}$.

Harmelink, D. J., J. E. Rowings. 1998. Linear scheduling model: Development of controlling activity path. Journal of Construction Engineering and Management 124(4): 263-268.

Harmelink, D. J. 2001. Linear scheduling model: Float characteristics. Journal of Construction Engineering and Management 127(4): 255-260.

Harris, R. B., P. G. Ioannou. 1998. Scheduling projects with repeating activities. Journal of Construction Engineering and Management 124(4): 269-278.

Kallantzis, A., S. Lambropoulos. 2004. Critical path determination by incorporating minimum and max- imum time and distance constraints into linear scheduling. Engineering, Construction and Architectural Management 11(3): 211-222.

Lucko, G. 2007a. Mathematical Analysis of Linear Schedules. Proceedings of the 2007 Construction Research Congress, eds. P. S. Chinowsky, A. D. Songer, P. M. Carrillo, Grand Bahama Island, The Commonwealth of the Bahamas, May 6-8, 2007, American Society of Civil Engineers, Reston, Virginia, $10 \mathrm{p}$.

Lucko, G. 2007b. Flexible modeling of linear schedules for integrated mathematical analysis. Proceedings of the 2007 Winter Simulation Conference, eds. Henderson, S. G., Biller, B., Hsieh, M.-H., Shortle, J., Tew, J. D., Barton, R. R., Washington, District of Columbia, December 9-12, 2007, Institute of Electrical and Electronics Engineers, Piscataway, New Jersey, 2159-2167.

O’Brien, J. J. 1975. VPM scheduling for high-rise buildings. Journal of the Construction Division, Proceedings of the American Society of Civil Engineers 101(CO4): 895-905.

Thabet, W. Y., Y. J. Beliveau. 1994. HVLS: Horizontal and vertical logic scheduling for multistory projects. Journal of Construction Engineering and Management 120(4): 875-892.

\section{AUTHOR BIOGRAPHIES}

GUNNAR LUCKO is Assistant Professor of Civil Engineering and Director of the Construction Engineering and Management Program in the Department of Civil Engineering at The Catholic University of America. He holds a German Diploma in Civil and Environmental Engineering from Hamburg University of Technology and an M.S. and $\mathrm{Ph} . D$. from Virginia Polytechnic Institute and State University (Virginia Tech). His research interests include mathematical representation, simulation, and analysis of schedule networks, construction equipment operations and economics, optimization methods, constructability analysis, and engineering education. He has studied statistical equipment valuation models and has participated in research for the Construction Industry Institute, and is currently working on research funded by the National Science Foundation. He is a member of ASCE and INFORMS.

ANGEL A. PEÑA OROZCO is a graduate research assistant in the Department of Civil Engineering at The Catholic University of America. He holds a B.S. in Industrial Engineering from Yacambú University in Venezuela and has over four years of project management experience in Venezuela and the U.S., where he worked on process reengineering, optimization, and business planning. He is currently performing research on schedule analysis with Dr. Gunnar Lucko. He is a student member of ASCE. 